\title{
D'une exploitation sectorielle à une exploitation territoriale
}

\author{
Perrine Vandenbroucke ${ }^{1}$, Jean Pluvinage ${ }^{2}$
}

\section{Introduction}

La transformation des exploitations agricoles, du sectoriel au territorial, pose question depuis la fin des années 1990 en Rhône-Alpes. Dans cette région à la fois montagneuse et périurbaine, les pouvoirs publics s'interrogent sur un renouvellement de la posture d'intervention publique afin de mieux prendre en considération le rôle structurant des activités agricoles pour le territoire régional. Les travaux pluridisciplinaires menés autour de la multifonctionnalité de l'agriculture constatent un renouvellement des rapports exploitations agricoles/territoires/filières de manière variable selon les espaces ${ }^{3}$. Ils conduisent ces chercheurs à faire l'hypothèse d'une divergence croissante entre des exploitations sectorielles inscrites dans une logique de compétitivité sur les marchés et des exploitations territoriales, pluriactives, multifonctionnelles et diversifiées.

Les notions d'exploitations sectorielles et territoriales, l'hypothèse est que cette évolution des "fonctions " de l'agriculture dans la société est associée à un processus de changement radical de ce qu'est l'unité de production agricole. Toutefois à une lecture duale simplificatrice entre grandes exploitations sectorielles et de petites exploitations diversifiées territoriales, nous considérons que la diversité des logiques des exploitations agricoles ne peut être lue qu'au regard de ce qui se joue dans les trois sphères que proposent C. Laurent et J. Rémy : ménage, secteur et territoire (Laurent et Rémy, 2000). Nous faisons l'hypothèse ici que l'avenir

1. ISARA-Lyon/Laboratoire d'études rurales, Agrapole, 69364 Lyon cedex 07, France, pvandenbroucke@isara.fr

2. Ancien directeur de recherche, INRA, département SAD, 7 Passage du Chemin Vert, 75011 Paris, France, jpluvinage@gmail.com

3. Réflexions pluridisciplinaires menées dans le cadre du programme « Pour et sur le développement régional "(PSDR, 2000-05) et dont est issu l'ouvrage collectif Nouvelles questions agraires. Exploitants, fonctions et territoires publiés par le laboratoire d'études rurales (LER) (Cornu, Mayaud, 2008).

Ce chapitre de l'ouvrage L'agriculture en famille : travailler, réinventer, transmettre est publié en Open Access sous licence creative commons CC-BY-NC-ND permettant l'utilisation non commerciale, la distribution, la reproduction du texte, sur n'importe quel support, à condition de citer la source. 
des exploitations agricoles se joue de plus en plus dans des territoires construits par des arrangements entre acteurs à l'échelle locale. Nous investiguons donc le territoire comme force de transformation de l'exploitation agricole. De ce fait, nous nous intéressons moins aux mutations liées aux évolutions intra-exploitation des ménages et des familles et aux mutations liées aux évolutions des rapports au sein des filières. En nous appuyant sur les travaux en économie de la régulation, secteur et territoire sont définis comme des niveaux d'organisation socioéconomiques intermédiaires où peut être défini l'avenir de l'exploitation agricole (Laurent et al., 2008). Selon cette approche, le secteur évoque les rapports de concurrence, les modalités d'organisation des échanges au sein des filières ainsi que les dispositifs sectoriels de politique agricole. Le territoire est défini comme un espace d'action collective ou d'action publique émergeant de la projection des groupes humains sur l'espace, et produit de leurs coordinations ou conflits dans la résolution d'un problème commun (Brunet et al., 1992 ; Raffestin, 1986) ${ }^{4}$. Cela renvoie plus spécifiquement aux dispositifs d'action publique territorialisés tels que les projets de territoire ou les dispositifs localisés de gestion de l'environnement. Il s'agit aussi de formes d'action collective localisées comme un groupe de producteurs locaux fédérés autour de la qualification territoriale d'un produit (Pecqueur, 2001). Enfin, sont parfois en jeu les arrangements et conflits relevant d'interactions localisées entre acteurs agricoles et non agricoles, autour de l'agrandissement d'une porcherie par exemple.

Un ensemble pluridisciplinaire de travaux met en lumière les termes de ce renouvellement de la relation de l'exploitation agricole au territoire, à partir de la fin des années 1990. Ces travaux constituent autant d'indicateurs d'un mouvement de (re)« territorialisation » de l'agriculture (Rieutort, 2009) tant en ce qui concerne l'insertion sociale des agriculteurs et l'évolution de leur métier (Le Caro et Kergreis, 2007), la définition des pratiques agricoles (Caron, 2005), et la définition des formes d'insertion marchande dans le cadre de systèmes agro-alimentaires localisés ou plus largement des systèmes agroalimentaires alternatifs (Deverre et Lamine, 2010). Cependant, la mise en place des contrats territoriaux d'exploitation (CTE) en région Rhône-Alpes aborde frontalement la question d'un " concernement " différencié des exploitations agricoles par les préoccupations territoriales (Bernard et al., 2006).

L'analyse présentée ici s'appuie sur les résultats d'un travail de thèse (Vandenbroucke, 2013). En croisant deux terrains d'étude, les Monts du Lyonnais et la Flandre intérieure, nous questionnons les formes de ce mouvement de territorialisation des exploitations agricoles. Notre hypothèse est que les exploitations agricoles se territorialisent plutôt dans des régions où elles sont fragilisées par les logiques sectorielles. Nous mettons en regard une région de moyenne montagne où l'avenir des exploitations laitières est fragilisé par la suppression des quotas; et une région agro-industrielle et périurbaine où la viabilité des exploitations n’est pas remise en

4. Les deux notions d'appropriation et de projection apparaissent ainsi centrales dans la définition du territoire. 
cause mais où le territoire s'impose comme une question incontournable. Le travail mobilise l'observation lors de manifestations publiques, l'analyse de différents documents (rapports d'étude, documents officiels, archives), et un corpus de 122 entretiens semi-directifs, avec des agriculteurs (62 entretiens) et avec différents acteurs des territoires (élus, techniciens des collectivités et organisations professionnelles agricoles, et acteurs associatifs).

Notre exposé procède en trois temps. Dans une première partie, nous mettons en perspective la genèse de ce questionnement sur un passage de logiques sectorielles à des logiques territoriales au regard des transformations de l'agriculture depuis 50 ans. Nous identifions ensuite dans les terrains étudiés les objets autour desquels se renouvelle le rapport exploitations agricoles-territoires dans des régions de polyculture-élevage et de proximité urbaine. Enfin, dans une troisième partie, nous tentons de dégager de cette analyse, certes partielle du fait du choix raisonné des deux terrains, une première typologie des exploitations agricoles selon trois idéaux types, qui déclinent à des degrés variables diverses formes de la territorialisation des exploitations agricoles.

\section{Un rapport renouvelé entre l'exploitation agricole et le territoire}

L'exploitation agricole est instituée dans les lois d'orientation de 1960-1962 comme une construction sociale et politique inscrite au sein du projet global d'organisation de la " production " agricole. La redéfinition de la place des producteurs comme maillons d'un système fordiste d'organisation de la production au sein de filières (Servolin et al., 1974), la consolidation identitaire d'un corps professionnel (Lémery, 2003), et l'accès privilégié au foncier dans des espaces ruraux dédiés à la fonction productive (Boinon, 2011) créent les conditions d'un modèle intégrateur d'insertion des exploitations agricoles dans le système économique et social. Par commodité pour la discussion, nous désignons cette forme canonique comme exploitation "sectorielle ", voulant exprimer ainsi le fait que l'avenir de ces exploitations se discute essentiellement entre acteurs du secteur agricole et agroalimentaire.

Or, dès le début des années 1980, les crises de surproduction, le coût de la politique agricole, la critique sociale émergente d'un modèle productiviste et la montée des préoccupations environnementales fragilisent ce modèle intégrateur (Müller, 1984). Celui-ci fait l'objet d'une contestation dans des réseaux en marge de l'organisation professionnelle, notamment dans les régions périphériques telles que les zones de montagne où émergent des modèles alternatifs, questionnés dans leur capacité à inscrire la logique de l'exploitation agricole dans le local. En montrant différentes formes de résistance de petites exploitations (Pernet, 1982) et le maintien de la pluriactivité (Lacombe, 1984), chercheurs et acteurs font ainsi émerger d'autres figures de l'agriculteur tel l'entrepreneur rural (Müller et al., 1989) ou les 
agriculteurs intermédiaires (Alphandéry et al., 1989). Néanmoins, le modèle de l'exploitation spécialisée n'est pas remis en cause de manière globale. Il se renforce dans une partition symbolique entre les exploitations professionnelles et les autres (Rémy, 1986).

Les débats sur la multifonctionnalité de l'agriculture autour de la loi d'orientation agricole (LOA) de 1999 et la mise en place des contrats territoriaux d'exploitation (CTE) (Brun, 2006) introduisent les termes d'un profond renouvellement du contrat social qui lie les agriculteurs à la société. Au gré des réformes successives de la politique agricole commune, nous assistons à un découplage avec les enjeux productifs et à un recouplage avec les enjeux environnementaux et du développement rural (Deverre et de Sainte Marie, 2008). De plus, la qualité s'impose comme le marqueur d'une transformation majeure des formes de régulation de l'agriculture et génère des opportunités de différenciation territoriale des produits de terroir par exemple via des dispositifs tels que les appellations d'origine contrôlée (Allaire et Boyer, 1995).

Par ailleurs, l'inversion démographique dans les espaces ruraux génère un renouvellement social et politique des espaces ruraux, et replace au cœur du débat la légitimité et les droits des différents acteurs sur l'espace et sur les ressources (Perrier-Cornet, 2002). Nous assistons à une «publicisation » de l'agriculture dont les objets sont mis en débat dans l'espace local par d'autres acteurs des territoires, élus, résidents, associations environnementales (Le Caro et Kergreis, 2007). Or, tant en matière de développement rural que d'action environnementale, un processus de territorialisation de l'action publique est engagé. Il est caractérisé par la relocalisation des échelles d'intervention et par un renouvellement des modalités d'action publique autour d'approches transversales multisectorielles et fondées sur le pari d'un partage d'une gouvernance de l'espace entre acteurs publics et privés (Berriet-Solliec et al., 2008). Le territoire s'affirme ainsi dans l'organisation politique comme l'instance de définition d'un " projet " tant en matière de développement économique, d'urbanisme ou de gestion des ressources. Cela contribue à l'émergence de nouvelles scènes de négociation entre les agriculteurs et d'autres acteurs de l'espace rural comme par exemple dans le cadre du dispositif Natura 2000 (Pinton et al., 2007).

Ainsi, l'exploitation agricole, désignée dans le code rural comme l'entreprise agricole et rurale depuis 2006, se situe donc aujourd'hui au carrefour de quatre grandes mutations : la redéfinition du contrat social entre les agriculteurs et la société dans le cadre des réformes successives de la politique agricole ${ }^{5}$ (1) ; un processus de publicisation de l'agriculture dans des espaces ruraux et périurbains où se confrontent logiques résidentielles, écologiques et productives (2); une redéfinition de la régulation du système agro-alimentaire et une demande sociale de qualité et de proximité qui génère des opportunités de différenciation territoriale des produits agricoles (3) ; et l'affirmation du territoire comme une composante structurante de

5. Sur ce point voir dans le même ouvrage, le chapitre rédigé par J. Pluvinage : L'exploitation agricole, entre famille et entreprise : 60 ans de débats et d'itinéraire de recherche personnel. 
l'espace politique, économique et social (4). L'exploitation agricole se trouve de ce fait profondément réinterrogée dans son rapport au territoire.

\section{Territorialisation de l'activité agricole dans les Monts du Lyonnais et en Flandre Intérieure}

\section{Deux petites régions de polyculture-élevage et de proximité urbaine}

Les Monts du Lyonnais constituent une zone de moyenne montagne, située entre les deux agglomérations de Lyon et Saint-Étienne. En dépit d'une périurbanisation à ses franges, cette région garde les caractéristiques d'un espace à dominante rurale avec une densité de population inférieure à $100 \mathrm{hab} . / \mathrm{km}^{2}$ et une part encore importante d'actifs agricoles et industriels. La structure agraire est concentrée autour de petites et moyennes exploitations (surface moyenne de 30 versus 53 hectares en France en 2010). Ces exploitations sont pour l'essentiel familiales (1,5 et 1,6 Unité Travail Agricole en moyenne par exploitation), avec cependant des tendances fortes d'évolution vers des formes sociétaires qui s'inscrivent de manière variable dans la continuité de ces exploitations familiales (Hervieu et al., 2013) : groupement agricole d'exploitation en commun (GAEC) à plusieurs associés, sociétés laitières, ou petites exploitations patronales diversifiées (un à cinq salariés). Alors que les exploitations laitières spécialisées et les exploitations à deux ateliers - un atelier herbivore et un atelier d'élevage ou de cultures intensives (porcs, volailles, fruits rouges, tabac) - représentaient jusqu'alors l'essentiel des exploitations, on assiste depuis 2000 à un relatif éclatement des structures : cessation laitière et spécialisation en fruits rouges ou en maraîchage, spécialisation d'exploitations en bovins viande. Les circuits courts constituent un vrai levier de pérennisation de ces petites et moyennes exploitations. Ils se développent dans de nouvelles formes d'organisation collectives (ateliers de transformation et points de vente collectifs) permettant de positionner les producteurs sur les marchés de Lyon et Saint-Étienne.

La Flandre intérieure constitue pour sa part une plaine argilo-limoneuse située entre les deux agglomérations de Lille et Dunkerque. Avec les deux petites villes d'Hazebrouck et Bailleul, cet espace est marqué par une histoire longue d'enchevêtrement du rural et de l'urbain. Depuis 1990, la périurbanisation de cette région située à 20 minutes de Lille génère un profond renouvellement de sa structure sociale et spatiale. Cette région qui présente un potentiel agricole élevé est caractérisée par la prédominante d'exploitations familiales et sociétaires sensiblement plus petites que la moyenne nationale ( 48 ha en moyenne au lieu de $53 \mathrm{ha}$ ). Une gamme de productions à forte valeur ajoutée - lait, porc, pommes de terre, cultures légumières et céréales - s'agence dans des combinaisons variables au sein des exploitations suivant les types de sols et l'histoire agraire. Trois configurations dominantes se dégagent : des exploitations sociétaires (GAEC ou SARL) à trois ateliers lait/porc/ pomme de terre; des exploitations familiales polyculture/lait dans les zones plus 
herbagères ; et des exploitations familiales ou sociétaires qui couplent porcs et pomme de terre dans les zones agricoles les plus denses. Plus ponctuellement, des exploitations spécialisées en polyculture ou des exploitations porcines hors-sol se sont développées dans des interrelations étroites avec leurs voisines (sous-location de terre pour assurer les rotations en pomme de terre, contrats de voisinage pour les droits d'épandage). Enfin, la vente directe et les activités de services (activités équestres notamment) se développent dans des logiques entrepreneuriales avec des salariés, et souvent individuelles. La structure agraire présente un caractère éclaté depuis de très petites exploitations pluriactives à de grandes exploitations spécialisées en polyculture (5-200 ha).

Dans ces régions à proximité de grandes agglomérations, le mouvement de périurbanisation transforme, à des degrés variables, l'organisation sociale et politique locale. Les agriculteurs sont confrontés à de nouvelles attentes sociétales autour de l'alimentation et de l'environnement parfois en décalage avec les dynamiques d'élevage intensif. Ainsi, dans un contexte d'évolution de la politique laitière, d'intégration croissante au sein des filières de polyculture et de pression environnementale sur la qualité de l'eau, les exploitations agricoles sont dans une phase de mutation dont nous questionnons la part des leviers territoriaux.

\section{Six objets autour desquels se renouvellent les rapports exploitation agricole-territoire}

Sur les terrains d'étude, Flandre intérieure et Monts du Lyonnais, ce renouvellement des rapports entre exploitations agricoles et territoires s'esquisse à partir de 1990 pour devenir une composante significative de l'évolution des exploitations agricoles à partir de 2000. Dans ces régions de polyculture-élevage et de proximité urbaine, les interactions entre les agriculteurs et les autres acteurs de l'espace local s'intensifient autour de six objets et scènes d'interaction :

1) la définition de la place et des fonctions de l'agriculture dans le projet de développement de ces territoires contribue soit à affirmer sa contribution à l'identité territoriale et cela renforce ainsi la place d'une élite agricole dans la dynamique de développement local dans les Monts du Lyonnais (Vandenbroucke, 2014) ; soit à ce que la collectivité affirme un modèle d'agriculture désirée, requalifiée autour de ses fonctions patrimoniales et de production de services (Lescureux, 2007), mais qui de fait s'inscrit en rupture avec la dynamique agricole locale ;

2) l'investissement patrimonial par une population urbaine des objets de l'agriculture génère pour les agriculteurs des opportunités de requalification identitaire et de valorisation de leurs produits et services dont ils se saisissent de manière variable. Ainsi, les fêtes et évènements agricoles (foires agricoles, fêtes des moissons, marché aux veaux...) très plébiscités deviennent des lieux de communication sur l'activité agricole. Les résidents s'approprient l'histoire agricole locale, les paysages et biens associés et créent ainsi des opportunités de 
différenciation territoriale des produits agricoles locaux tel par exemple le houblon des Flandre via la qualification de bières régionales ;

3) la requalification des liens de proximité entre producteurs et consommateurs autour de la question de l'alimentation contribue à une réorganisation des circuits de commercialisation. Dès 1980, on voit se dégager sur ces deux territoires, qui ont toujours été marqués par ces rapports de proximité villecampagne, une évolution de la clientèle, de ses attentes, des gammes de produits commercialisés et des formes de distribution. La vente directe se professionnalise, les producteurs s'organisent autour de points de vente collectifs, et de nouvelles formes d'organisation marchande émergent (associations des producteurs d'une commune pour l'approvisionnement local de la restauration collective, mise en réseau avec des associations de consommateurs urbains) ;

4) les dispositifs territorialisés de gestion des ressources naturelles contribuent à mettre en débat les pratiques agricoles dans des scènes de négociations qui impliquent d'autres acteurs. La gestion des quantités de nitrates et de produits phytosanitaires dans les eaux de surface dans les Monts du Lyonnais s'affirme ainsi comme une composante d'une dynamique renouvelée d'innovation et de changement technique (Vandenbroucke et Pluvinage, 2010). Les problématiques de régulation des inondations en Flandre suscitent des micro-conflits locaux dont émergent souvent des aménagements de pratiques concertés entre agriculteurs, collectivités et riverains ;

5) la hausse de la conflictualité entre agriculteurs et leur voisinage liée à la concentration dans les élevages bovins et porcins dans la région Nord-Pasde-Calais place l'activité agricole au cœur d'une controverse locale et conduit les agriculteurs à se repositionner, individuellement et collectivement, par rapport à leur environnement social de proximité ;

6) les enjeux de partage du foncier et de gestion des concurrences entre activités quant à l'usage de l'espace conduisent les agriculteurs à se positionner sur des scènes de négociations locales dans l'élaboration des documents d'urbanisme (Plan local d'urbanisme - PLU - et Schéma de cohérence territoriale - SCOT), ou lors d'aménagement d'infrastructures urbaines ou écologiques.

Ces six objets présentent une certaine généricité typique à ces espaces au carrefour entre les territorialités du résidentiel, de l'écologie et des activités de production. Comme illustré ci-dessus, ce redéploiement des interrelations entre les agriculteurs et les autres acteurs de ces espaces génère cependant de manière variable une dynamique de territorialisation des exploitations agricoles sur ces deux terrains.

Dans les Monts du Lyonnais, ces objets partagés sont les supports d'arrangements institutionnels construits par l'action collective et l'action publique dans une perspective de redéfinition des conditions de viabilité des exploitations agricoles sur cet espace. Ce processus de territorialisation s'inscrit dans l'histoire longue de la consolidation identitaire d'un collectif agricole et d'une communauté politique territoriale. Il est caractérisé par l'intégration progressive dans la définition des orientations 
productives et socio-structurelles de différentes variables liées à la recomposition sociale de cet espace rural.

En Flandre intérieure, la multiplication des situations de conflits autour de ces objets révèle la mise en tension d'un espace entre les logiques productives, résidentielles et environnementales. À l'exception de quelques filières territorialisées, nous n'identifions pas un processus de territorialisation des exploitations agricoles, mais une logique d'adaptation individuelle des agriculteurs à l'évolution de leur contexte social et spatial par la contrainte ou par l'opportunité.

\section{Trois idéaux-types de territorialisation des exploitations agricoles}

Au-delà des particularités propres à chaque situation territoriale ainsi identifiée, trois idéaux-types se dégagent de manière transversale de nos deux terrains d'étude bien que présents dans des proportions variables (Tab. 1). Ces idéaux-types donnent une représentation des processus socioéconomiques qui se déroulent dans les interactions exploitations agricoles-territoires. Nous employons cette notion d'idéaux-types pour illustrer l'idée qu'il s'agit de polarités entre lesquelles se situent les exploitations agricoles. Il ne s'agit pas de définir les critères d'un classement des exploitations agricoles. Ces trois idéaux-types présentent une certaine généricité et peuvent être envisagés comme opérants pour penser l'action publique et le développement de l'agriculture face à une interrogation centrale que celle de l'articulation entre les logiques de filières et les logiques territorialisées d'aménagement et de protection de l'environnement et de gestion des ressources. Pour chacun de ces idéauxtypes, nous pouvons identifier certaines régularités de positionnement individuel des agriculteurs. Nous déclinons donc à un niveau plus fin les registres d'action mobilisés par les agriculteurs concernés. 


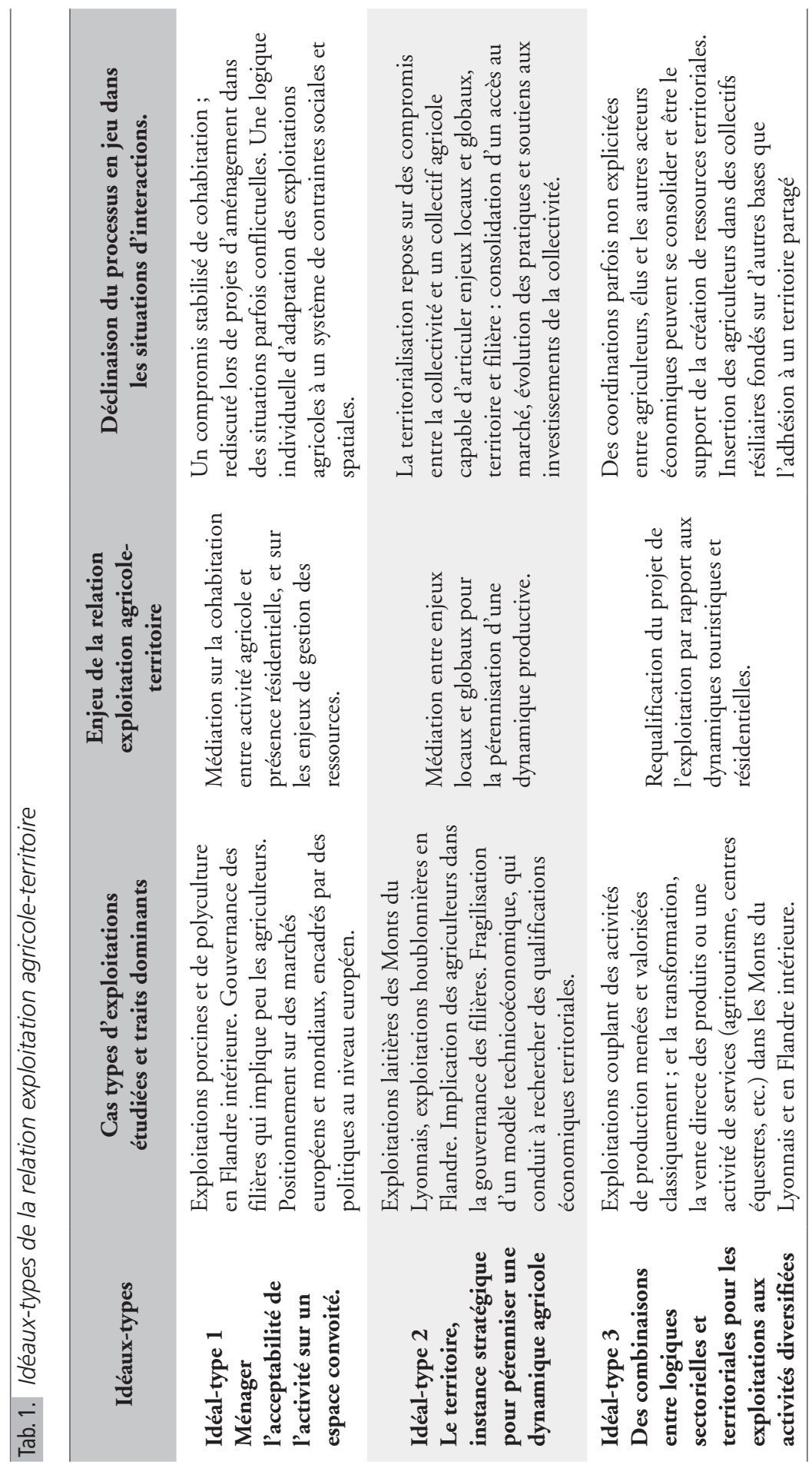




\section{Idéal-type 1 : ménager l'acceptabilité de l'activité agricole sur un espace convoité}

Un premier idéal-type désigne les exploitations agricoles qui acceptent un minimum d'aménagements à effectuer pour ménager l'acceptabilité de l'activité sur un espace donné, vis-à-vis d'enjeux de cohabitation ou de gestion des ressources. Il s'agit d'exploitations agricoles caractérisées par leur insertion dans un système agro-industriel au sein duquel les agriculteurs sont peu impliqués dans la définition des cahiers des charges, avec un positionnement sur des marchés européens et mondiaux. Cet idéal-type apparaît particulièrement représenté dans notre étude en Flandre intérieure, pour le cas des exploitations agricoles porcines ou de polyculture industrielle (céréales, légumes de conserverie, pommes de terre) (Photo 1).

Dans ces zones d'élevage de Flandre et des Monts du Lyonnais, les agriculteurs sont impliqués dans l'entretien des chemins ruraux, et des ajustements de leurs pratiques d'élevage (enfouissement des épandages). Il existe un système implicite de règles de bonnes pratiques de cohabitation dont la définition se déroule à l'échelle de la commune. Ces ajustements individuels de pratiques assurent la pérennisation dans le temps long de l'exploitation agricole sur cet espace (Nicourt et al., 2006). Mais en Flandre où la contiguïté spatiale est forte, ce compromis stabilisé est remis en cause lors d'enquêtes publiques pour l'agrandissement des élevages porcins ; lors de projets d'aménagements routiers ; ou dans la résolution de problèmes concrets tels que des inondations. Ces négociations, souvent conflictuelles, conduisent les agriculteurs à effectuer des aménagements de pratiques, telle par exemple l'installation de haies en bordure de parcelle. Ces aménagements se déclinent toutefois à la marge du fonctionnement du système de production. Les agriculteurs privilégient une logique d'externalisation de la gestion des enjeux environnementaux par des compromis de partage de l'espace.

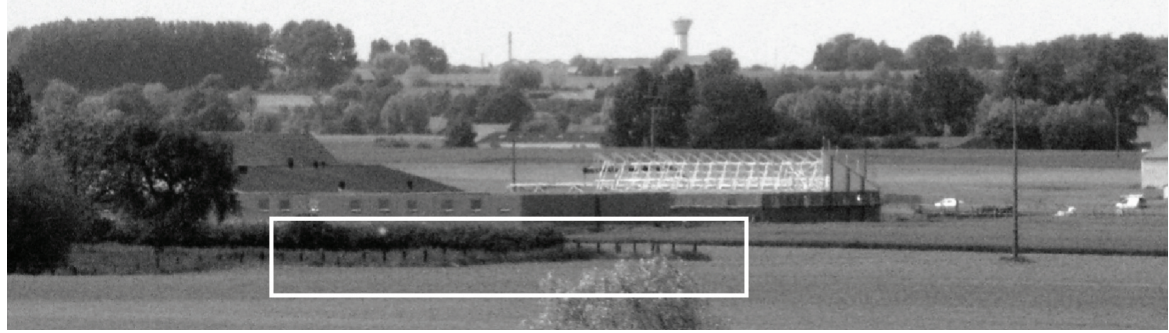

Photo 1. Aménagements paysagers pour rendre acceptable l'agrandissement d'une porcherie (C P. Vandenbroucke, Flandre intérieure, 23 juin 2010)

Les registres d'action mobilisés individuellement par les agriculteurs dans ce premier idéal-type relèvent soit d'une logique défensive par rapport aux contraintes d'un 
territoire subi ; soit d'une logique d'anticipation et de négociation par une implication dans le jeu social et politique locale, la communication et des aménagements de pratiques. Les agricultrices jouent souvent un rôle de médiatrices dans l'espace social local soit par leur implication dans la vie communale et associative, soit par le développement d'activités pédagogiques permettant de communiquer sur leur activité. Mais dans certains cas, cette logique poussée à l'extrême s'apparente à une approche industrielle de gestion des risques liés à l'activité dans la stratégie productive et relationnelle.

Pour conclure, il n'y a pas pour ce premier idéal-type de changement de la nature des instances où se joue l'avenir de ces exploitations agricoles, mais une logique d'adaptation individuelle à des enjeux locaux qui s'imposent de manière incontournable autour de questions environnementales ou de gestion de la proximité résidentielle. En revanche, ces adaptations individuelles peuvent sur le long terme définir les ingrédients d'une réorientation de la trajectoire de l'exploitation agricole sous l'effet d'un resserrement du système de contraintes ou de la saisie d'opportunités. La perspective d'une retraite prochaine et la recherche de nouvelles sources de revenus, l'installation d'un jeune, ou d'une conjointe sur l'exploitation avec leurs propres projets tels le développement des activités équestres ou de la vente directe, sont les ingrédients premiers de ces bifurcations. Avec des parcours de vie et de formation à l'extérieur (moniteur équestre, institutrice...), un autre rapport au travail par rapport aux contraintes d'un élevage par exemple, conjoints et enfants d'agriculteurs peuvent mettre en perspective le potentiel de développement d'une exploitation agricole au regard non seulement des dynamiques sectorielles, mais aussi d'autres ressources et opportunités latentes telles que la situation géographique en bord de route touristique ou l'existence d'un marché de proximité des produits et services.

Ces exploitations sont donc faiblement territorialisées parce que leur activité les inscrit dans d'autres espaces et systèmes de contraintes, mais elles restent des entreprises locales à même de changer de trajectoires et ainsi requalifier partiellement leur rapport au territoire, au travers de la diversité des activités des membres de familles d'agriculteurs.

\section{Idéal-type 2 : le territoire, instance stratégique pour pérenniser une dynamique agricole}

Un second idéal-type regroupe dans notre analyse les exploitations agricoles pour lesquelles le territoire est investi comme une instance stratégique pour pérenniser une dynamique productive. Les planteurs de houblon en Flandre et les producteurs laitiers des Monts du Lyonnais sont particulièrement représentatifs de cet idéal-type. Le territoire se construit autour d'un collectif agricole local qui crée des alliances tant au sein des instances sectorielles qu'au niveau des instances territoriales pour définir les conditions de la pérennisation d'un système productif.

Trois conditions apparaissent essentielles à l'inscription des exploitations agricoles dans de telles démarches : la présence d'un collectif d'agriculteurs capable 
d'articuler les questions territoriales et les enjeux de la filière, l'existence d'un espace sociotechnique local autour d'une production, et la fragilisation d'un modèle technico-économique. Les exploitations de cet idéal-type 2 se distinguent de l'idéal-type 1 car des institutions locales sont mises en jeu dans la gouvernance de la filière via l'implication des agriculteurs dans le cadre de coopératives de production, via la présence de négociants locaux qui travaillent en partenariat étroit avec les agriculteurs ou via des dispositifs tels que les quotas par lesquels les producteurs locaux sont parties prenantes de la gouvernance.

La territorialisation repose sur l'action centrale d'un collectif d'agriculteurs fédéré autour d'une identité forte autour du produit ou du métier et appuyé par différentes organisations professionnelles et des collectivités qui s'impliquent à divers titres (emploi, paysage, patrimoine...) pour le maintien de cette dynamique productive. Trois profils d'agriculteurs apparaissent particulièrement impliqués dans ce processus : des responsables professionnels "stratèges » et multi-casquettes; des agriculteurs innovateurs qui anticipent sur les limites d'un modèle technique ; et des agriculteurs " innovateurs sociaux » qui conçoivent différemment leur métier dans les relations aux acteurs non agricoles et au mouvement social et s'impliquent localement dans une démarche militante.

Les difficultés économiques, telle la fragilisation de la filière laitière des Monts du Lyonnais depuis 2003, sont un moteur d'une implication proactive des agriculteurs dans les démarches territoriales avec la perspective d'y consolider des ressources pour pérenniser la dynamique productive. La territorialisation se traduit concrètement par un appui des collectivités territoriales au financement d'infrastructures individuelles et collectives, et par l'émergence de nouveaux espaces d'expérimentation et d'innovation technique qui intègrent les enjeux locaux. Ainsi, dans les Monts du Lyonnais, la récente halle aux veaux permettant le maintien d'un marché aux veaux naissants important tant pour les agriculteurs que pour la collectivité a été entièrement financée sur des fonds publics. Cela se traduit également par des compromis sur les changements de pratiques par rapport à des enjeux locaux de qualité de l'eau à travers la contractualisation ou l'expérimentation collective. Ces engagements territoriaux négociés localement s'inscrivent aussi dans une stratégie portée au niveau de la filière laitière régionale à la recherche de ressources pour maintenir de la production dans ces espaces de moyenne montagne. Des stratégies analogues peuvent être décryptées pour la micro-filière houblonnière en Flandre à la suite d'une crise majeure dans le courant des années 1980.

Ainsi, pour cet idéal-type 2 nous identifions bien une dynamique de territorialisation des exploitations agricoles. Celle-ci repose sur l'implication d'agriculteurs et d'agricultrices en activité. Le territoire peut être caractérisé comme un espace de médiation entre les enjeux socio-économiques d'une dynamique agricole locale, d'enjeux environnementaux ou économiques locaux portés par la collectivité, et des dynamiques plus larges dans lesquelles s'inscrivent les exploitations agricoles (politique agricole, etc.). 


\section{Idéal-type 3 : des combinaisons entre logiques sectorielles et territoriales pour les exploitations aux activités diversifiées}

L'idéal-type 3 regroupe des exploitations agricoles qui couplent une activité de production et des activités de services ou de vente directe relocalisée ou en relation avec les villes voisines.

Ces démarches de diversification des activités dans une exploitation relèvent d'abord de trajectoires personnelles et familiales : réponse à des situations de crise, installation, positionnement personnel au sein de la famille. Dans ce contexte de changement, les familles requalifient leurs opportunités de positionnement sur un marché local des produits et services. Le projet de l'exploitation est ainsi resitué par rapport aux dynamiques touristiques et résidentielles.

Ces évolutions ne sont pas neutres, elles conduisent à modifier les relations de l'agriculteur avec les autres acteurs locaux, notamment dans la relation marchande. $\mathrm{Si}$ certains cas de coordination collective forte peuvent être identifiés dans les Monts du Lyonnais par exemple pour l'approvisionnement d'un restaurant scolaire, les logiques des agriculteurs sont souvent individuelles et parfois concurrentes entre elles, notamment en Flandre par rapport à une clientèle locale. Même si les agriculteurs ne sont pas forcément à l'origine de démarches collectives reposant sur l'initiative des collectivités (marques collectives, par exemple), ils contribuent à des formes de territorialisation souvent non explicitées autour de ressources territoriales partagées telles que l'image d'un territoire, ou un paysage (Photo 2). Ils contribuent à l'attractivité touristique globale en désignant un fromage par le nom d'un lieudit. Ils bénéficient en retour des actions mises en place par d'autres pour faire venir une clientèle sur le territoire (évènementiel, chemins de randonnée). Cela ne prend cependant pas forme dans une coordination explicitée entre ces acteurs.

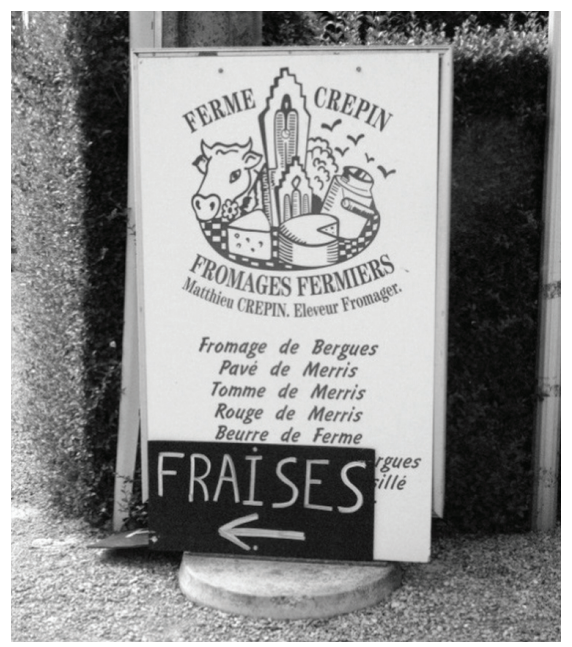

Photo 2. L'image d'un territoire saisie comme ressource territoriale (C) P. Vandenbroucke, Flandre intérieure, 23 juin 2010). 
Le développement de ces activités contribue à rattacher les agriculteurs à des collectifs externes, souvent résiliaires, qui reposent sur d'autres bases que la référence à un territoire partagé. Ainsi, un agriculteur biologique en réseau avec les associations de consommateurs telles que les Associations pour le maintien d'une agriculture paysanne est isolé du collectif agricole local, mais inséré dans les réseaux citoyens et militants des agglomérations voisines.

On retrouve ainsi parmi les exploitations agricoles qui se rapprochent de cet idéaltype une grande diversité de combinaisons entre le sectoriel, le territorial et d'autres institutions telles que l'adhésion à une éthique partagée.

\section{Conclusion}

Pour conclure, ce travail nous permet de mettre en évidence qu'il se dégage clairement un mouvement de territorialisation qui concerne toutes les exploitations agricoles, mais qui n'exprime une évolution réelle de celles-ci que dans certaines configurations. De plus, il apparait simplificateur d'opposer le secteur au territoire dans les dynamiques en cours. Les transformations observées des exploitations agricoles se traduisent surtout dans des combinaisons variables entre logiques sectorielles et territoriales. Nous distinguons en particulier trois idéaux-types : un premier idéal-type d'exploitations très sectorielles et peu territoriales; un second idéal-type caractérisé par un couplage fort des logiques sectorielles et territoriales par l'intermédiaire d'un collectif sociotechnique local ; et un troisième idéaltype d'exploitations agricoles diversifiées qui combinent de manière variable les dimensions sectorielles, territoriales et insérées dans des configurations résiliaires non directement territoriales. Ces trois idéaux-types rendent compte de situations observées pour les exploitations agricoles sur deux terrains de polyculture-élevage et de proximité urbaine. Ils ne sont pas exhaustifs des différentes formes de la territorialisation et leur portée générique reste questionnée dans d'autres types d'espaces, en particulier dans des régions de plus faible densité telles que la Beauce ou le Massif central, ou bien de régions plus rurales, comme l'Ouest. On peut faire l'hypothèse que la proportion d'exploitations se rattachant à tel ou tel idéal-type varie en fonction de la configuration historique et géographique, mais également que d'autres idéaux-types se dégagent.

En mettant en perspective les dynamiques des exploitations agricoles entre ces idéaux-types, cette analyse nous permet de souligner le rôle des collectifs, agricoles et non agricoles, dans la dynamique de territorialisation. Les collectifs agricoles locaux jouent un rôle central de territorialisation par la médiation entre les enjeux locaux et la dynamique productive. En ce sens, la création des groupements d'intérêts économiques et écologiques (GIEE) dans le cadre de la loi d'avenir pour l'agriculture, l'alimentation et la forêt prévue en 2014 pourrait être un moteur de territorialisation des exploitations agricoles. Mais, sont également impliqués dans cette dynamique de territorialisation les collectifs non agricoles, qui, selon leurs configurations locales ou résiliaires, contribuent à élargir les sphères socio-économiques 
dans lesquelles s'inscrivent les exploitations agricoles sans qu'il y ait une démarche volontaire des agriculteurs en ce sens. Cela repose la question de la posture des acteurs par rapport à ces dynamiques de territorialisation de l'économie.

Nos enquêtes nous permettent également d'observer que les familles des agriculteurs peuvent jouer un rôle dans la médiation entre les logiques résidentielles et la dynamique agricole et dans la requalification du potentiel de l'exploitation agricole par rapport à celles-ci. Toutefois, cette question n'était pas l'objet central de nos enquêtes. Cela ne nous permet donc pas d'en faire un traitement systématique et reste à réserver pour des investigations ultérieures.

\section{Bibliographie}

Allaire, G., Boyer, R., 1995. La grande transformation de l'agriculture : lectures conventionnalistes et régulationnistes, Paris, INRA.

Alphandéry, P., Bitoun, P., Dupont, Y., 1989. Les champs du départ : une France rurale sans paysans? Paris, La découverte.

Bernard, C., Dobromez, L., Pluvinage, J., Dufour, A., Havet, A., Mauz, I., Pauthenet,Y., Rémy, J., Tchakérian, E., 2006. La multifonctionnalité à l'épreuve du local : les exploitations agricoles face aux enjeux des filières et des territoires, Cahiers Agricultures, 15, 6, 523-528.

Berriet-Solliec, M., Deprés, C., Trouvé, A., 2008. La territorialisation de la politique agricole en France. Vers un renouvellement de l'intervention publique en agriculture ? , in Laurent, C., Du Tertre, C., Secteurs et territoires dans les régulations émergentes, Paris, L'Harmattan, 121-136.

Boinon, J.-P., 2011. Les politiques foncières agricoles en France depuis 1945, Économie et statistique, 444-445, 19-37.

Brun, G., 2006. L'agriculture française à la recherche d'un nouveau modèle, Paris, L'Harmattan.

Brunet, R., Ferras, R., Thery, H., 1992. Les mots de la géographie : dictionnaire critique, Paris, La documentation française.

Caron, P., 2005. À quels territoires s’intéressent les agronomes? Le point de vue d'un géographe tropicaliste, Natures Science Sociétés, 13, 2, 145-153.

Cornu, P., Mayaud, J.-L., 2008. Nouvelles questions agraires. Exploitants, fonctions et territoires, Paris, La boutique de l'histoire.

Deverre, C., de Sainte Marie, C., 2008. L'écologisation de la politique agricole européenne. Verdissement ou refondation des systèmes agro-alimentaires ? Revue d'études en agriculture et environnement, 89, 4, 83-104.

Deverre, C., Lamine, C., 2010. Les systèmes agroalimentaires alternatifs. Une revue de travaux anglophones en sciences sociales, Économie rurale, 317, 57-73.

Hervieu, B., Purseigle, F., 2013. Sociologie des mondes agricoles, Paris, Armand Colin. 
Lacombe, P., 1984. La pluriactivité et l'évolution des exploitations agricoles, in Association des Ruralistes Français, in La pluriactivité dans les familles agricoles, Paris, ARF Editions, 35-53.

Laurent, C., Du Tertre, C., Dieuaide, P., Petit, P., 2008. Introduction. Régulations sectorielles et territoriales au coeur du nouveau régime d'accumulation, in Laurent, C., Du Tertre, C., Secteurs et territoires dans les régulations émergentes, Paris, L'Harmattan.

Laurent, C., Rémy, J., 2000. L'exploitation agricole en perspective, Courrier de l'environnement, 41, 5-24.

Le Caro, Y., Kergreis, S., 2007. L'espace agricole comme espace public : accès récréatif et entretien du bocage en Bretagne, in Le Caro, Y. , Madeline, P., Pierre, G., Agriculteurs et territoires. Entre productivisme et exigences territoriales, Rennes, Presses Universitaires de Rennes, 231-250.

Le Caro, Y., Madeline, P., Pierre, G., 2007. Agriculteurs et territoires. Entre productivisme et exigences territoriales, Rennes, Presses Universitaires de Rennes, Espace et Territoires.

Lémery, B., 2003. Les agriculteurs dans la fabrique d'une nouvelle agriculture, Sociologie du travail, 45, 1, 9-25.

Lescureux, F., 2007. L'agriculture désirée dans les pays du Nord-Pas-de-Calais, in Le Caro, Y., Madeline, P., Pierre, G., Agriculteurs et territoires. Entre productivisme et exigences territoriales, Rennes, Presses Universitaires de Rennes, 87-103.

Mormont, M., 2006. Conflit et territorialisation, Géographie Économie Société, 8, 3, 299-318.

Müller, P., 1984. Le technocrate et le paysan. Essai sur la politique française de modernisation de l'agriculture, de 1945 à nos jours, Paris, Éditions de l'Atelier.

Müller, P., Faure, A., Gerbaux, F., 1989. Les Entrepreneurs ruraux : agriculteurs, artisans, commerçants, élus locaux, Paris, L'Harmattan.

Nicourt, C., Girault, J.-M., 2006. Une co-construction territoriale des règles du travail d'éleveur, Économie rurale, 291, 42-59.

Pecqueur, B., 2001. Qualité et développement territorial : l'hypothèse du panier de biens et de services territorialisés, Économie rurale, 261, 37-49.

Pernet, F., 1982. Résistances paysannes, Grenoble, Presses universitaires de Grenoble.

Perrier-Cornet, P., 2002. À qui appartient l'espace rural ? La Tour d'Aigues, co-éditions de l'Aube/DATAR.

Pinton, F., Alphandéry, P., Billaud, J.-P., Deverre, C., 2007. La Construction du réseau Natura 2000 en France : une politique publique européenne à l'épreuve du terrain, Paris, La Documentation Française.

Raffestin, C., 1986. Ecogénèse territoriale et territorialité, in Auriac, F., Brunet, R., Espace, jeux et enjeux, Paris, Fayard, 173-185.

Rémy, J., 1986. Profession : agriculteur, Paris, INRA.

Rieutort, L., 2009. Dynamiques rurales françaises et re-territorialisation de l'agriculture, L'information géographique, 73, 1, 30-48. 
Servolin, C., Gervais, M., Nallet, H., Coulomb, P., 1974. L'agriculture dans le système social-recueil d'articles, Paris, INRA.

Vandenbroucke, P., 2013. Transformation de l'unité de production agricole, d'une exploitation sectorielle à une exploitation agricole territoriale. Exploitations agricoles, agriculteurs et territoires dans les Monts du Lyonnais et en Flandre intérieure de 1970 à 2010, Thèse de Géographie, Aménagement et Urbanisme, Laboratoire d'études rurales, Université Lyon 2.

Vandenbroucke, P., 2014. Le maintien d'une élite agricole au coeur de la définition des orientations territoriales dans les Monts du Lyonnais (1970-2010), in Sarrazin, F., Les élites agricoles et rurales. Concurrences et complémentarités des projets, Rennes, Presses Universitaires de Rennes, 95-214.

Vandenbroucke P. et Pluvinage J., 2010. Transformation of fodder systems in the "Monts du Lyonnais» : interactions between farmers and territorial stakeholders, ISDA, Innovation and Sustainable Development in Agriculture, Montpellier, 28 juin- $1^{\text {er }}$ juillet. 